\title{
Cefaleia associada a compressão do terceiro nervo craniano por um aneurisma da artéria comunicante posterior
}

\author{
Headache associated with compression of the third cranial nerve by an \\ aneurysm of the posterior communicating artery
}

\begin{abstract}
Marcelo M. Valença ${ }^{1,2}$, Joacil Carlos da Silva ${ }^{1,2}$, Marcos Barbosa ${ }^{1,2}$, Martina F. Valença ${ }^{3}$, Laryssa Azevedo Almeida , Maria Carolina M. Oliveira', Cássia L. S. França ${ }^{7}$, Raíza R. B. Oliveira ${ }^{7}$, Míriam C. Soares', Eduardo C. Nunes ${ }^{7}$, Rafael G. Costa ${ }^{1}$, Helysândia S. S. Farias ${ }^{7}$, Isabela S. Saraiva', Arthur C. Holanda', Válter R. Souza Junior', Mário L. M. Silva Júnior', Maria Carolina Cavalcanti Lima', Marília Apolinário Batista', Paloma P. Travassos', Ulyscélio S. M. Ferreira', Maria de Fátima V. Aragão', Luciana P. A. Andrade Valença ${ }^{1,2}$

'Grupo de Pesquisa Avanços em Neurocirurgia, Universidade Federal de Pernambuco, Recife, Brasil ${ }^{2}$ Serviço de Neurocirurgia, Hospital Esperança, Recife, Brasil 3University of Basel, Basel, Switzerland
\end{abstract}

\begin{abstract}
Valença MM, Silva JC, Barbosa M, Valença MF, Almeida LA, Oliveira MC, França CL, Oliveira RR, Soares MC, Nunes EC, Costa RG, Farias HS, Saraiva IS, Holanda AC, Souza Junior VR, Silva Júnior ML, Lima MC, Batista MA, Travassos PP, Ferreira US, Aragão MF, Valença LP. Cefaleia associada a compressão do terceiro nervo craniano por um aneurisma da artéria comunicante posterior. Headache Medicine. 2014;5(1):21-24
\end{abstract}

\begin{abstract}
Continuous unilateral pain in the orbital area may be a sign of an intracranial structural lesion. We report a case of a 64 -yearold woman with continuous headache of moderate intensity at the region of the left eye bulb. There was a complete paralysis of the left third oculomotor nerve. The patient was admitted with headache, vomiting, palpebral ptosis, divergent strabismus of the left eye, diplopia when looking to the left, upwards and downwards and paralytic mydriasis on the left. The Angiography revealed a saccular aneurysm dilation in the internal carotid artery with origin at the origin of the left posterior communicating artery. A microsurgery was performed on the aneurysm of the left internal carotid artery and the aneurysm was occluded by clipping. On four months follow up the patient is asymptomatic, with a complete recovery of the third nerve palsy. There is still much controversy about microsurgical and endovascular therapy in cerebral aneurysms, this case represents the good outcome in a patient with paralysis of the oculomotor nerve after microsurgery occlusion of a posterior communicating aneurysm. In conclusion, all patients with a continuo pain in the orbital area need to be evaluated by angiography in order to display a intracranial aneurysm.
\end{abstract}

Keywords: Anaurysm, third cranial nerve, headache, pain, treatment

\section{RESUMO}

Dor unilateral contínua na área orbital pode ser um sinal de uma lesão estrutural intracraniana. Relatamos o caso de uma mulher de 64 anos de idade, com dor de cabeça contínua, de intensidade moderada na região do olho esquerdo. Havia uma completa paralisia do nervo oculomotor esquerdo. A paciente foi internada com dor de cabeça, vômitos, ptose palpebral, estrabismo divergente do olho esquerdo, diplopia ao olhar para a esquerda, para cima e para baixo e midríase paralítica do lado esquerdo. A angiografia revelou um aneurisma sacular na artéria carótida interna com origem na saída da artéria comunicante posterior esquerda. A microcirurgia foi realizada e o aneurisma foi ocluído com um clipe. Em quatro meses do acompanhamento a paciente está assintomática, com uma recuperação completa do déficit do terceiro nervo craniano. Ainda há muita controvérsia sobre como devemos tratar um aneurisma cerebral, se por abordagem microcirúrgica ou por via endovascular. Neste artigo apresentamos o caso de uma paciente com uma boa recuperação da paralisia do nervo oculomotor, após a oclusão microcirurgia de um aneurisma da comunicante posterior. Em conclusão, todos os pacientes com dor contínua na região da órbita têm de ser avaliados por angiografia, a fim de se excluir ou confirmar a presença de um aneurisma cerebral como causa da dor. 
Palavras-chaves: Aneurisma, terceiro nervo craniano, cefaleia, dor, tratamento

\section{INTRODUÇÃO}

Dor na região da órbita de caráter contínuo, principalmente quando tem início recente (e.g. poucos dias), é um sinal de alerta (red flag) indicando a possibilidade de haver uma lesão estrutural intracraniana como causa desse sintoma. Uma das possibilidades é a presença de aneurismas cerebrais comprimindo estruturas que podem provocar dor no indivíduo. Quando a dor é acompanhada por paralisia do terceiro nervo craniano, ou seja, porque o paciente apresenta ptose palpebral progressiva, visão dupla e estrabismo divergente, devemos aventar a possibilidade da presença de um aneurisma da artéria carótida interna, com origem na saída da artéria comunicante posterior, com localização no mesmo lado da dor e da ptose palpebral.(1) Isso é particularmente importante quando há anisocoria com midríase no lado onde a dor é referida. A midríase é por conta da compressão, pelo aneurisma, sobre as fibras parassimpáticas que correm na periferia do nervo oculomotor. ${ }^{(1)}$

O propósito deste artigo é relatar um caso de uma paciente com dor ocular associada com paralisia completa do terceiro nervo craniano que após o procedimento cirúrgico, com oclusão do aneurisma com uso de clipe, houve recuperação do déficit oculomotor.

\section{RELATO DO CASO}

Mulher com 64 anos de idade, com história de dor no globo ocular esquerdo há uma semana, de moderada intensidade, contínua, porém com flutuação na intensidade durante o dia. Procurou uma emergência médica que não valorizou o sintoma. Sintomáticos foram prescritos e a paciente foi encaminha para sua residência. No sétimo dia surgiu ptose palpebral esquerda e, uma hora após quando se dirigia para ser atendida em uma emergência médica, apresentou, ainda no seu carro, uma cefaleia "em trovoada", ou seja, súbita e intensa acompanhada por vômito. A paciente era hipertensa e negava passado de tabagismo ou história familiar de aneurisma cerebral.

Ao exame a paciente estava orientada, lúcida, cooperava com o exame, comunicava-se normalmente pela palavra falada, nuca livre, força normal nos quatro membros. Na avaliação neurológica foi encontrada como anormalidade apenas uma paralisia completa do terceiro nervo craniano esquerdo. Havia ptose palpebral completa (Figura 1), estrabismo divergente do olho esquerdo, diplopia no olhar conjugado para esquerda, para cima e para baixo quando a pálpebra esquerda era manualmente levantada. Observou-se também midríase paralítica esquerda.

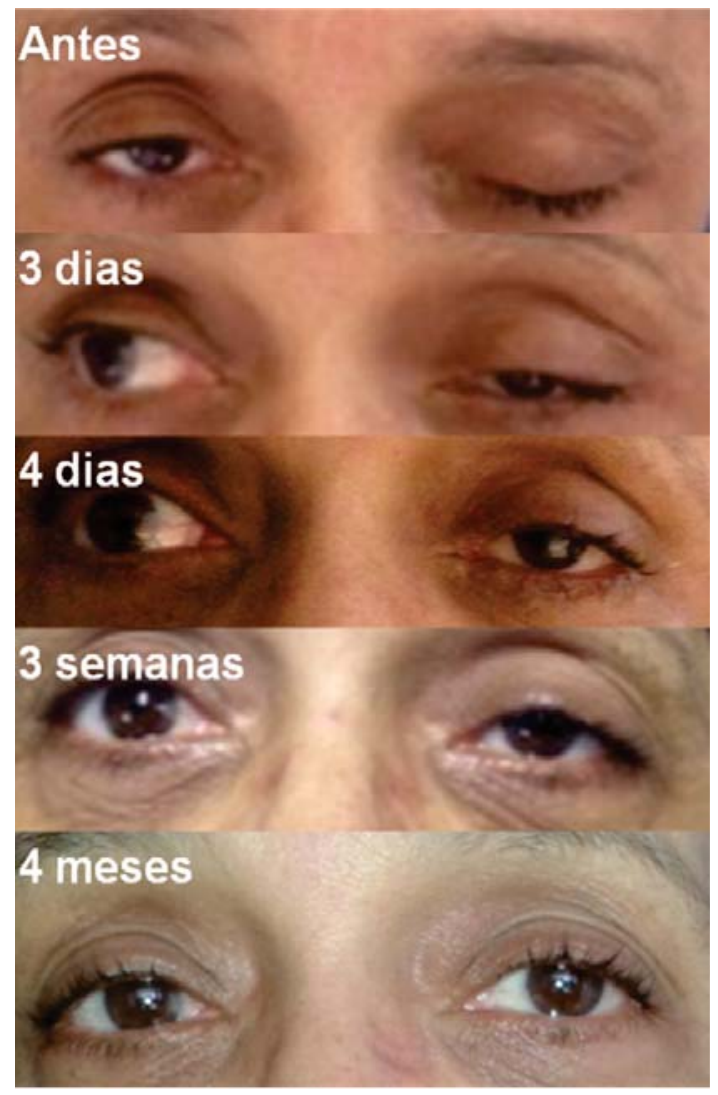

Figura 1. Recuperação da ptose palpebral esquerda após clipagem microcirúrgica de um aneurisma da artéria carótida internacomunicante posterior esquerda ao longo de 4 meses.

Uma tomografia computadorizada foi considerada normal, sem qualquer sinal de hemorragia subaracnoidea. No mesmo dia foi submetida a uma angiografia cerebral, quando uma dilatação aneurismática sacular foi identificada na artéria carótida interna, com origem na saída da artéria comunicante posterior esquerda, com características de ruptura, com colo curto e o saco aneurismático orientado para baixo e para esquerda, medindo em seus maiores eixos 5,65 mm x 3,85 mm e com o colo medindo 2,50 mm (Figura 2). Havia ausência de vasoespasmo. Um outro aneurisma foi identificado na origem da artéria cerebelar póstero-inferior (PICA) na artéria vertebral direita (Figuras 3 e 4). 


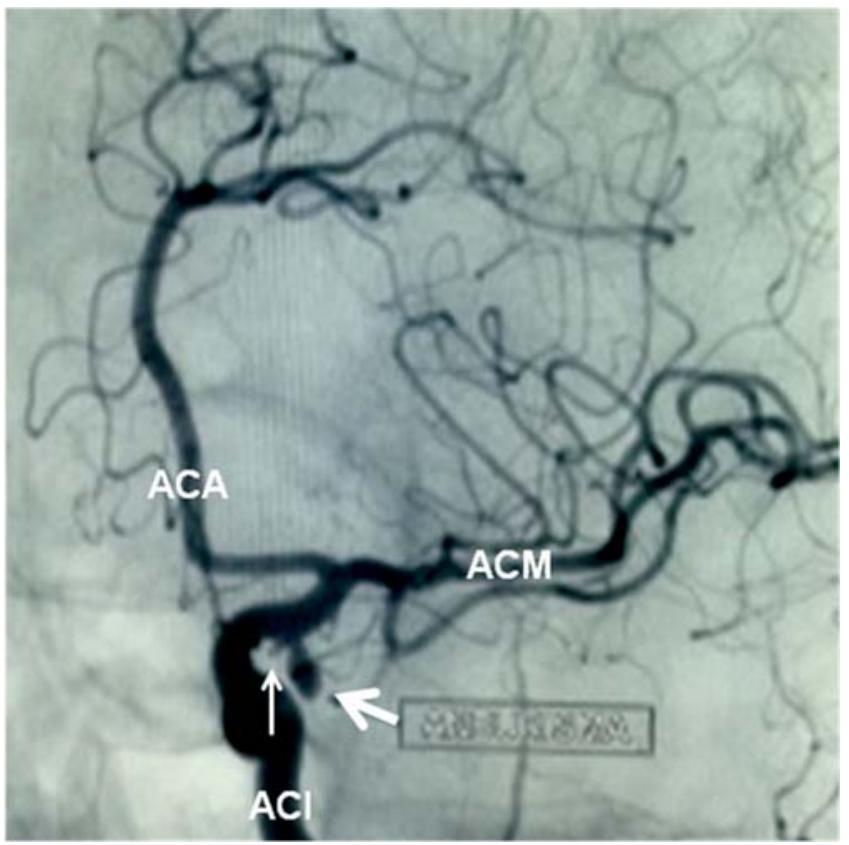

Figura 2. Angiografia cerebral mostrando um aneurisma sacular (seta maior) da artéria carótida interna $(\mathrm{ACl})$, na origem da artéria comunicante posterior esquerda (seta menor). ACM, artéria cerebral média; $A C A$, artéria cerebral anterior padrão ázigo.

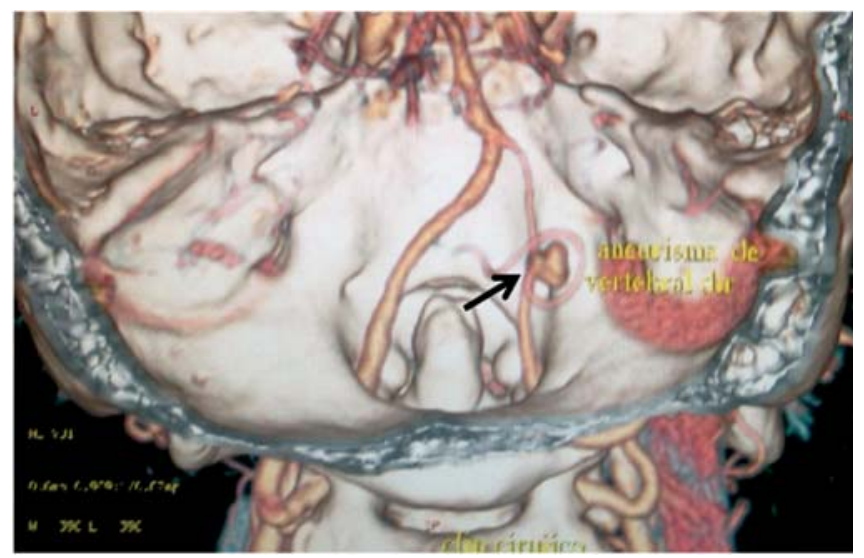

Figura 3. Ângio-TC mostrando um aneurisma não roto (seta) na saída da artéria cerebelar póstero-inferior (PICA) em relação à artéria vertebral direita.

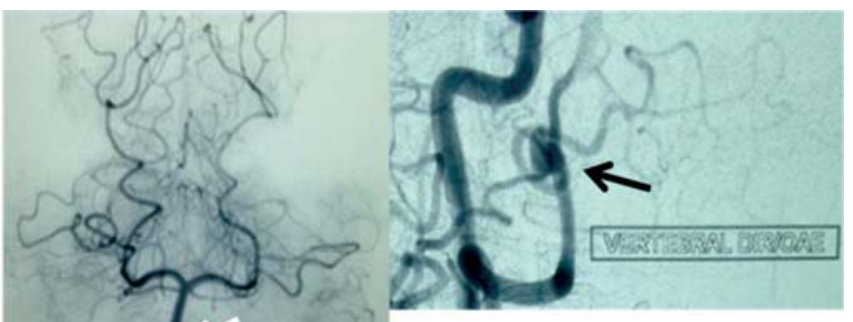

Figura 4. Estudo angiográfico mostrando fenestração da artéria basilar (seta branca) e a presenca de um aneurisma na artéria vertebral direita, na origem da PICA (seta preta).
Como variações anatômicas foram encontradas: (1) fenestração da artéria basilar no seu segmento proximal (Figura 4) e (2) artéria cerebral anterior do tipo ázigo (Figura 2).

Havia duas possibilidades para oclusão do aneurisma da artéria carótida interna-comunicante posterior esquerdo - por via endovascular ou através de uma abordagem microcirúgica com uso de clipes.(2-4) Optou-se pelo procedimento microcirúrgico pela maior possibilidade de recuperação da paralisia do terceiro nervo craniano, segundo evidências publicadas recentemente. ${ }^{(3-4)}$

No dia seguinte à sua admissão a paciente foi submetida a um procedimento microcirúrgico com clipagem do aneurisma da artéria carótida interna esquerda, por via pterional esquerda com remoção da clinóide anterior esquerda e uso de um clipe em baioneta de titânio.

No pós-operatório imediato a dor cedeu e três dias após o procedimento cirúrgico notou-se já uma recuperação parcial da ptose palpebral (Figura 1). Quatro meses após a oclusão do aneurisma e consequente descompressão do terceiro nervo craniano houve regressão completa da ptose palpebral (Figura 1).

Três semanas após o procedimento cirúrgico um novo estudo angiográfico foi realizado, mostrando oclusão completa do aneurisma da artéria carótida interna esquerda. A posição do clipe em relação à carótida é mostrada na Figura 5.

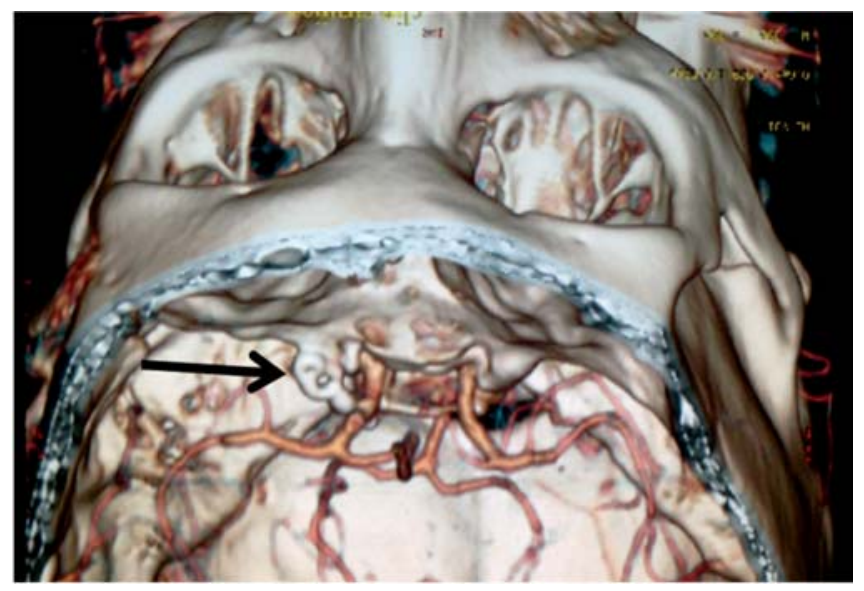

Figura 5. Ângio-TC realizada no pós-operatória da paciente mostrando o bom posicionamento do clipe (seta preta), bem próximo da base do crânio, com oclusão completa do aneurisma.

Quatro meses depois do primeiro procedimento a paciente teve seu aneurisma da PICA ocluído por via endovascular.

A paciente encontra-se assintomática, orientada e sem déficit neurológico focal. 


\section{COMENTÁRIOS}

Ainda há muita controvérsia sobre a melhor forma de ocluir um aneurisma intracraniano, se por via endovascular ou através de um acesso microcirúrgico.(2-4) Contudo, parece que um consenso está sendo estabelecido no sentido de que quando há compressão de nervos intracranianos o melhor seria uma abordagem microcirúrgica, possibilitando um descompressão direta da estrutura neural. ${ }^{(3-4)}$

No caso particular do terceiro nervo craniano a clipagem direta do aneurisma parece ser a melhor opção, como foi o caso aqui relatado. $O$ cirurgião não deve, entretanto, separar o saco aneurismático no nervo oculomotor, porque isso pode danificar ainda mais essa estrutural neural. Se achar que haveria algo a mais a fazer, isso seria separar o restante do saco aneurismático (deixando ele aderido ao nervo) do colo que encontrase clipado, usando-se uma microtesoura.

Güresir e colegas ${ }^{(3)}$ encontraram 10 em 344 (2,9\%) aneurismas intracranianos que causavam paralisia do terceiro nervo craniano. Esses autores estudaram 201 aneurismas da artéria comunicante posterior (132 pacientes foram submetidos a clipagem microcirúrgica, 54 pacientes foram tratados por via endovascular, e 15 pacientes não foram tratados). Na análise multivariada, clipagem cirúrgica foi significativamente associada com resolução parcial de qualquer grau da paralisia $(O R 12,2,95 \% \mathrm{Cl}$ 3-49) e de resolução completa (OR, 7, 1, 95\% Cl 1,8-28) em comparação com pacientes com tratamento endovascular. Indicando a superioridade do procedimento microcirúrgico em relação ao endovascular para esse tipo particular de aneurisma cerebral.

Estudo recente ${ }^{(5)}$ mostra que o tamanho do aneurisma pode não influenciar o aparecimento da ptose palpebral, mas a proximidade da carótida das estruturas da base do crânio, incluindo o terceiro nervo, parece ser mais importante como valor associativo. No nosso caso, a clinoide anterior teve de ser removida para possibilitar a clipagem adequada do aneurisma, mostrando uma localização baixa do aneurisma, bem próximo da base do crânio, com compressão do nervo por um aneurisma relativamente pequeno (menor do que $6 \mathrm{~mm}$ ).

Outros aspectos clínicos e relacionados com a fisiopatogenia da compressão do oculomotor por um aneurisma são revisados em publicação anterior. ${ }^{(6)}$

Concluíndo, todos os pacientes com dor ocular fixa, que perdura por vários dias, devem ser avaliados por neuroimagem, incluindo ângio-ressonância magnética ou angiotomografia computadorizada, mesmo aqueles sem o acometimento da pupila, cuja possibilidade maior seria acometimento do terceiro nervo craniano por uma mononeuropatia diabética ou isquêmica, na qual as fibras parassimpáticas do oculomotor estão poupadas.

\section{REFERÊNCIAS}

1. Valença MM, Andrade-Valença LPA, Martins C. Functional anatomy of headache: circle of Willis aneurysms, third cranial nerve and pain. Headache Medicine. 2011 ;2(3):82-88

2. Gu DQ1, Luo B, Zhang X, Long XA, Duan CZ. Recovery of posterior communicating artery aneurysm-induced oculomotor nerve paresis after endovascular treatment. Clin Neurol Neurosurg. 2012 Nov; $114(9): 1238-42$. doi: 10.1016/i.clineuro. 2012.03.016. Epub 2012 Mar 29.

3. Güresir E, Schuss P, Setzer M, Platz J, Seifert V, Vatter H. Posterior communicating artery aneurysm-related oculomotor nerve palsy: influence of surgical and endovascular treatment on recovery: single-center series and systematic review. Neurosurgery. 2011 Jun;68(6):1527-33; discussion 1533-4. doi: 10.1227/NEU. Ob013e31820edd82.

4. Güresir E, Schuss P, Seifert V, Vatter H. Oculomotor nerve palsy by posterior communicating artery aneurysms: influence of surgical strategy on recovery. J Neurosurg. 2012 Nov; $117(5): 904-10$. doi: 10.3171/2012.8.JNS1 1 1239. Epub 2012 Aug 31.

5. Anan M, Nagai Y, Fudaba H, Kubo T, Ishii K, Murata K, Hisamitsu Y, Kawano Y, Hori Y, Nagatomi H, Abe T, Fujiki M. Third nerve palsy caused by compression of the posterior communicating artery aneurysm does not depend on the size of the aneurysm, but on the distance between the ICA and the anterior-posterior clinoid process. Clin Neurol Neurosurg. 2014 Aug;123: 169 73. doi: 10.1016/i.clineuro.2014.05.006. Epub 2014 Jun 4.

6. Valença MM, Andrade-Valença LP, Martins C, de Fátima Vasco Aragão M, Batista LL, Peres MF, da Silva WF. Cluster headache and intracranial aneurysm. J Headache Pain. 2007 Oct;8(5):277-82. Epub 2007 Oct 23.

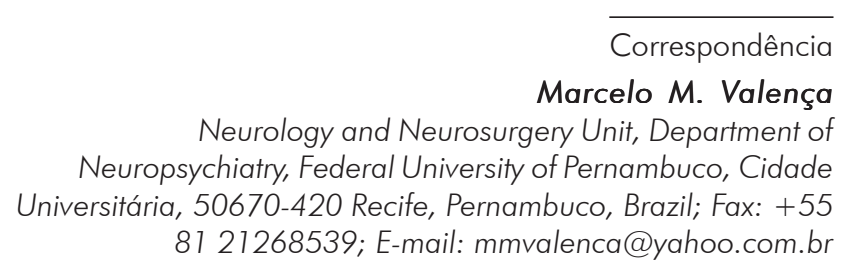

Received Dec 5, 2013

Accepted Jan 25, 2014 